\title{
Number, position, and significance of the pseudouridines in the large subunit ribosomal RNA of Haloarcula marismortui and Deinococcus radiodurans
}

\author{
MARK DEL CAMPO, ${ }^{1}$ CLAUDIA RECINOS, ${ }^{2}$ GISCARD YANEZ, ${ }^{2}$ STEVEN C. POMERANTZ, ${ }^{3}$ \\ REBECCA GUYMON, ${ }^{3}$ PAMELA F. CRAIN, ${ }^{3}$ JAMES A. MCCLOSKEY, ${ }^{3,4}$ and JAMES OFENGAND ${ }^{2,5}$ \\ ${ }^{1}$ Institute for Cellular and Molecular Biology, University of Texas at Austin, Austin, Texas 78712, USA \\ ${ }^{2}$ Department of Biochemistry and Molecular Biology, University of Miami School of Medicine, Miami, Florida 33101, USA \\ ${ }^{3}$ Department of Medicinal Chemistry and ${ }^{4}$ Department of Biochemistry, University of Utah, Salt Lake City, Utah 84112, USA
}

\begin{abstract}
The number and position of the pseudouridines of Haloarcula marismortui and Deinococcus radiodurans large subunit RNA have been determined by a combination of total nucleoside analysis by HPLC-mass spectrometry and pseudouridine sequencing by the reverse transcriptase method and by LC/MS/MS. Three pseudouridines were found in $H$. marismortui, located at positions 1956, 1958, and 2621 corresponding to Escherichia coli positions 1915, 1917, and 2586, respectively. The three pseudouridines are all in locations found in other organisms. Previous reports of a larger number of pseudouridines in this organism were incorrect. Three pseudouridines and one 3-methyl pseudouridine $\left(\mathrm{m}^{3} \Psi\right)$ were found in $D$. radiodurans $23 S$ RNA at positions 1894, $1898\left(\mathrm{~m}^{3} \Psi\right), 1900$, and 2584 , the $\mathrm{m}^{3} \Psi$ site being determined by a novel application of mass spectrometry. These positions correspond to $E$. coli positions $1911,1915,1917$, and 2605 , which are also pseudouridines in $E$. coli (1915 is $\mathrm{m}^{3} \Psi$ ). The pseudouridines in the helix 69 loop, residues 1911, 1915, and 1917, are in positions highly conserved among all phyla. Pseudouridine 2584 in D. radiodurans is conserved in eubacteria and a chloroplast but is not found in archaea or eukaryotes, whereas pseudouridine 2621 in $\mathrm{H}$. marismortui is more conserved in eukaryotes and is not found in eubacteria. All the pseudoridines are near, but not exactly at, nucleotides directly involved in various aspects of ribosome function. In addition, two $D$. radiodurans $\Psi$ synthases responsible for the four $\Psi$ were identified.
\end{abstract}

Keywords: pseudouridine sequencing; $H$. marismortui; $D$. radiodurans; mass spectrometry sequencing; pseudouridine synthases

\section{INTRODUCTION}

Pseudouridine $(\Psi)$ (Cohn 1960) is the most common modified nucleoside found in RNA (Rozenski et al. 1999). Although it was first identified in a precise position in the TYCG sequence in tRNA (Zamir et al. 1965) and later at other sites (Sprinzl and Vassilenko 2002), many more $\Psi$ residues are to be found in ribosomal RNA, both in the large and small rRNAs (Maden 1990, Ofengand and Bakin 1997; Ofengand et al. 2001b; Ofengand 2002). The determination of the sites of the $\Psi$ residues in large ribosomal subunit (LSU) RNAs of a number of representative species

${ }^{5}$ Deceased December 6, 2004.

Reprint requests to: Mark Del Campo, Lambowitz Laboratory, Institute for Cellular and Molecular Biology, University of Texas at Austin, 1 University Station A4800, Austin, TX 78712, USA; e-mail: mdelcamp_mail@ yahoo.com; fax: (512) 232-3420.

Article and publication are at http://www.rnajournal.org/cgi/doi/ 10.1261/rna.7209905. has revealed a remarkable conservation of location, although not necessarily of exact position. $\Psi$ in small ribosomal subunits do not appear to specifically localize to known functional sites except for Escherichi coli, whose single $\Psi$ is near the decoding site (Ofengand et al. 2001b; Ofengand 2002). In E. coli, the LSU $\Psi$ cluster in and around the peptidyl transferase center (PTC), here loosely defined as that region that binds the amino acyl ends of tRNAs in the $\mathrm{A}, \mathrm{P}$, and $\mathrm{E}$ sites. There is also a set of three in the terminal loop of helix 69. This stem-loop is thought to interact with $\mathrm{A}$ and $\mathrm{P}$ site bound tRNAs (Yusupov et al. 2001). Despite this juxtaposition to functional regions of the LSU, the significance of the locations of $\Psi$ has remained obscure.

Localization of the $\Psi$ residues in a known three-dimensional ribosome structure could be expected to help define $\Psi$ function. Unfortunately, those organisms whose ribosomal $\Psi$ have been mapped (Ofengand and Bakin 1997) have not had their ribosome structures determined, and the 
two organisms whose LSU structure is known, Haloarcula marismortui (Ban et al. 2000) and Deinococcus radiodurans (Harms et al. 2001), have not had their rRNA mapped for $\Psi$. This study was initiated to remedy this lack of knowledge. Here we report the $\Psi$ content and sites for the 23S RNA of $H$. marismortui and D. radiodurans.

\section{RESULTS}

\section{Determination of the total number of $\Psi$}

As a prelude to, and a reality check on, mapping the $\Psi$ sites by sequencing, the total number of $\Psi$ in the ribosomal RNAs of $D$. radiodurans and $H$. marismortui were determined (Table 1). The methodology was checked by analysis of $E$. coli rRNA, whose number of $\Psi$ sites is known from complete sequence analysis (Ofengand 2002). As shown in the table, values close to expected were

A

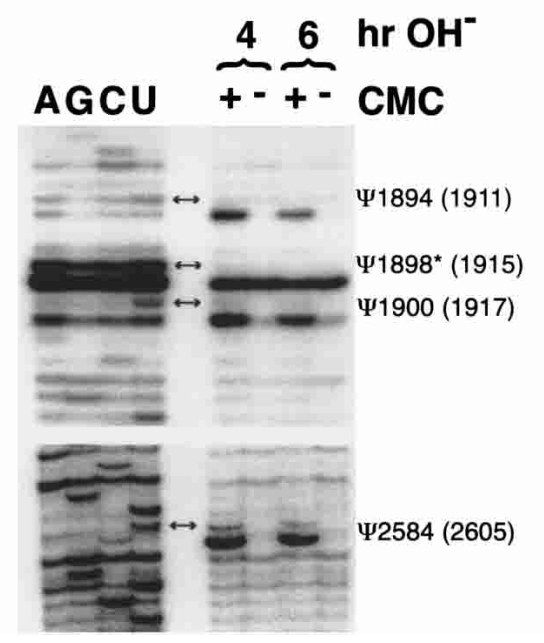

B

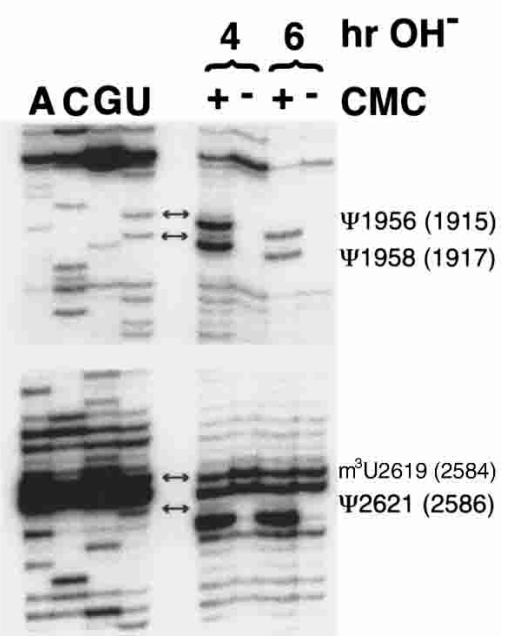

FIGURE 1. $\Psi$ sequencing analysis of 23 S RNA from D. radiodurans $(A)$ and H. marismortui $(B)$. RNA was treated with $(+)$ or without $(-)$ CMC followed by treatment with alkali $\left(\mathrm{hr} \mathrm{OH}^{-}\right)$for 4 or $6 \mathrm{~h}$, and then used as template for primer extension with reverse transcriptase (Ofengand et al. 2001a). In this assay, a CMC-dependent reverse transcription stop one base $3^{\prime}$ of a uridine (indicated by arrows) identifies a $\Psi$. The position of each $\Psi$ is numbered and $E$. coli equivalents are given in parentheses. RNA sequence is shown in $\mathrm{A}, \mathrm{C}, \mathrm{G}$, and $\mathrm{U}$ lanes. $\left(^{\star}\right)$ Putative $\mathrm{m}^{3} \Psi$. The autoradiographs shown are the only $\Psi$ found in two comprehensive analyses covering residues 1-2852 (99\%) of D. radiodurans and residues 1-595, 633-2887 (98\%) of H. marismortui $23 \mathrm{~S}$ RNA. The stop in all lanes in $B$ corresponding to $\mathrm{m}^{3} \mathrm{U} 2619$ is indicated.

obtained. Earlier analysis of the accu-

racy of the total digestion method (Gehrke and Kuo 1990) in our hands suggested errors of $\pm 15 \%$ of the number of modified residues measured (Noon et al. 1998). The slight discrepancy in the 23S RNA value could thus be due to measurement error and/or $<100 \%$ modification at one or more of the known sites. There was no detectable $\Psi$ in $H$. marismortui 16S RNA but three $\Psi$ were found in the $23 \mathrm{~S}$ RNA. This value agrees with the sequence analysis (see Fig. 1 ), indicating that virtually complete modification had occurred at each site. There was no detectable $\Psi$ in $D$. radiodurans 16S RNA whereas the 23S RNA had a measured net value of 2.0 residues. Since three $\Psi$ sites were clearly identified by sequencing (see below), it appears that in this case the average modification level of the $\Psi$ sites was only $67 \%$.

TABLE 1. Total number of pseudouridines in $\mathrm{H}$. marismortui and D. radiodurans ribosomal RNA

\begin{tabular}{lcc}
\hline & 16S RNA & 23S RNA \\
\hline E. coli & $1.1(1)$ & $8.1\left(9^{\mathrm{a}}\right)$ \\
H. marismortui & $<0.1$ & 3.1 \\
D. radiodurans & $<0.1$ & 2.0 \\
\hline
\end{tabular}

Analysis was done as described in Materials and Methods. Values in parentheses are those expected for $E$. coli based on sequencing analysis (Ofengand 2002).

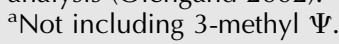

\section{Mapping the location of $\Psi$ in $D$. radiodurans and $H$. marismortui 23S RNA}

To precisely locate the positions of 23S RNA $\Psi$, total RNA from $D$. radiodurans and $H$. marismortui was isolated and subjected to $\Psi$ sequencing. Comprehensive analysis of $99 \%$ of the $D$. radiodurans 23S RNA sequence located three $\Psi$ at positions U1894, U1900, and U2584 (Fig. 1A); the CMCindependent stop at U1898 will be addressed below. Similarly, three $\Psi$ were located in $H$. marismortui $23 \mathrm{~S}$ RNA at positions U1956, U1958, and U2621 (Fig. 1B). This total number of $\Psi$ sites is consistent with the amount obtained by RNase digestion and HPLC analysis (Table 1).

\section{Other modifications}

Methylation or other modification that blocks base-pair formation has the effect of resulting in a pause or stop of reverse transcription, resulting in a band across all lanes of a sequencing gel. We could confirm the finding by Hansen et al. (2002) of a strong reverse transcriptase stop at U2619 (2584) as shown in Figure 1B. We also observed a very strong stop in several independent preparations of $H$. marismortui 23S RNA at A629 with stuttering at A628 (data not shown). These results are consistent with the finding of $\mathrm{m}^{3} \mathrm{U} 2619$ and $\mathrm{m}^{1} \mathrm{~A} 628$ by X-ray crystallography (Klein et al. 2004). The RNAs from both D. radiodurans and H. marismortui were more fragmented than typically found in other organisms (Ofengand and Bakin 1997). Therefore, it was not possible to assign the additional bands that occurred 
across all lanes as sites of putative modification with any degree of certainty.

\section{Identification and location of 3-methylpseudouridine $\left(m^{3} \Psi\right)$ in $D$. radiodurans 235 RNA}

Any $\Psi$ with an additional modification (such as a methyl group) that interferes with base pairing would produce a CMC-independent stop of reverse transcriptase and thus could not be confirmed as $\Psi$ since the assay is based on the CMC dependence of reverse transcriptase blockade. This is the case for $\Psi 1915$ in E. coli, which was shown to be $\mathrm{m}^{3} \Psi$ (Kowalak et al. 1996). In that case, a CMC-independent stop for $\Psi 1915$ appeared between the CMC-dependent stops for $\Psi 1911$ and $\Psi 1917$. However, $\Psi 1915$ was produced upon reaction of a $23 \mathrm{~S}$ RNA transcript with its purified $\Psi$ synthase in vitro (Raychaudhuri et al. 1998). An identical pattern was observed for the equivalent residues in $D$. radiodurans $23 \mathrm{~S}$ RNA. There is a single CMC-independent stop for U1898 between the CMC-dependent stops for $\Psi 1894$ and $\Psi 1900$ (Fig. 1A). This observation implied that U1898 might also be $\mathrm{m}^{3} \Psi$. This was established by LC/MS analysis of a total rRNA nucleoside digest, after first determining the complete absence of this nucleoside in the $16 \mathrm{~S}$ portion of the RNA. The diagnostic mass spectral peaks and exact HPLC elution times were directly compared in backto-back analyses against $E$. coli $23 \mathrm{~S}$ rRNA that contains $\mathrm{m}^{3} \Psi$ (Kowalak et al. 1996). In both experiments the elution time of the putative $\mathrm{m}^{3} \Psi$ component (11.2 $\mathrm{min}$ ) was accurately matched against that of $\mathrm{m}^{5} \mathrm{C}$ as an internal retention time marker, using $m / z 259\left(\mathrm{MH}^{+}\right.$for $\mathrm{m}^{3} \Psi$ and the first isotope peak from $\mathrm{MH}^{+}$of $\mathrm{m}^{5} \mathrm{C}$ ) and $\mathrm{m} / z$ 223, the characteristic $\mathrm{MH}^{+}-2 \mathrm{H}_{2} \mathrm{O}$ ion of any methylpseudouridine. Peaks for $\mathrm{m} / z$ 259 and $\mathrm{m} / \mathrm{z} 223$ from reconstructed ion chromatograms were co-incident in time as required for the $\mathrm{m}^{3} \Psi$ nucleoside, and followed the $\mathrm{m} / z 259$ peak for $\mathrm{m}^{5} \mathrm{C}$ by $3.6 \mathrm{sec}$ in both rRNA analyses (data not shown). This result confirmed the presence of $\mathrm{m}^{3} \Psi$ in $D$. radiodurans $23 \mathrm{~S}$ rRNA.

To determine if $\mathrm{m}^{3} \Psi$ is present in D. radiodurans $23 \mathrm{~S}$ RNA specifically at position 1898 , an aliquot of the mixed $16 \mathrm{~S}$ and 23S RNA was digested with RNase T1 and subjected to LC/MS/MS analysis in the negative ion mode for de novo sequencing ( $\mathrm{Ni}$ et al. 1996) of the predicted oligonucleotide 1894- $\Psi \mathrm{AACm}^{3} \Psi \mathrm{A} \Psi \mathrm{AACGp}$ (Fig. 2). Ions were observed in the low mass region of the CID spectrum that are consistent with the presence of a methylpseudouridine residue. These included ions of $m / z 417,337$, and 319, corresponding in mass to a methyluridine diphosphate, monophosphate, and its dehydration product, respectively (not annotated in Fig. 2). The presence of methylpseudouridine, and not a uridine methylated in the base or ribose, was deduced by the observation of an ion at $\mathrm{m} / z 221$ (not annotated; the negative ion counterpart of $223^{+}$discussed above), which is consistent with the interpretation of $m \Psi$ $2 \mathrm{H}_{2} \mathrm{O}$, the facile loss of water being a hallmark of pseudo-

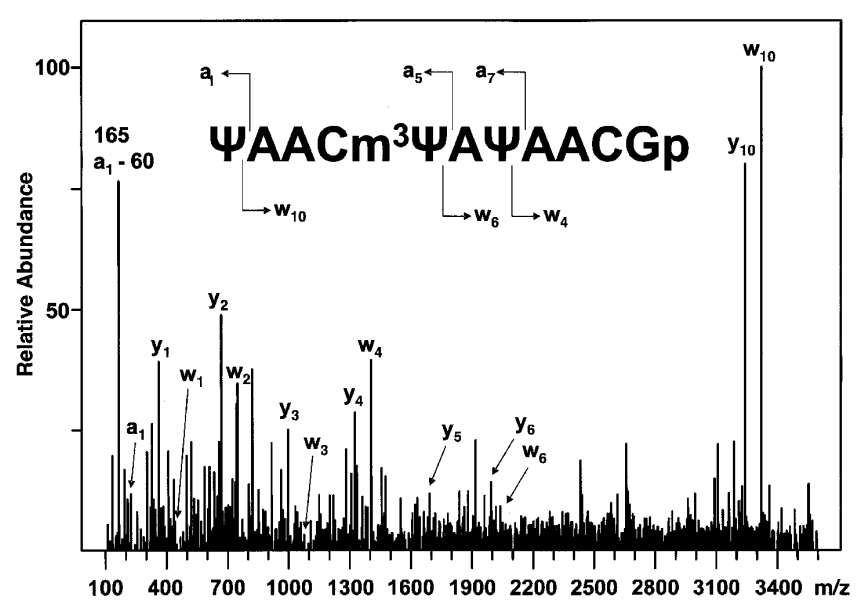

FIGURE 2. Sequencing mass spectrum of RNase T1 fragment $M_{r}$ 3550.5 from $D$. radiodurans LSU RNA showing $\mathrm{m}^{3} \Psi$ at position 1898 . The spectrum is represented in maximum entropy deconvolution (MaxEnt3) format.

uridine mass spectra (e.g., Felden et al. 1998). No ions characteristic of a base-methylated uridine $(\mathrm{m} / \mathrm{z} 125)$, which would have been indicative of a normal N-C glycosidic bond, were observed. The methyl group was localized to position 1898 by the nearly complete series of $\mathbf{w}$ and $\mathbf{y}$ series sequencing ions listed in Table 2 (for sequence ion nomenclature see McLuckey et al. 1992). These ions are indicative of the sequence from the $3^{\prime}$ terminus, whose interpretation necessitated the presence of a methyl group in the fifth nucleotide (corresponding to rRNA position 1898). The mass difference of 320 units between ions $\mathbf{w}_{\mathbf{6}}$ and $\mathbf{w}_{\mathbf{7}}$ (Table

TABLE 2. Sequence ions from the mass spectrum of $D$. radiodurans RNase T1 product $M_{r} 3550.5^{a}$ (Fig. 2)

\begin{tabular}{|c|c|c|}
\hline Assignment & Mass, calc. & Mass, found \\
\hline$y_{1}$ & 362.050 & 362.061 \\
\hline$y_{2}$ & 667.091 & 667.105 \\
\hline$y_{3}$ & 996.144 & 996.174 \\
\hline$y_{4}$ & 1325.196 & 1325.222 \\
\hline$y_{5}$ & 1631.222 & 1631.217 \\
\hline$y_{6}$ & 1960.274 & 1960.292 \\
\hline$y_{7}$ & 2280.319 & 2280.343 \\
\hline$y_{8}$ & 2585.360 & 2585.466 \\
\hline$y_{9}$ & 2914.413 & $2914.712^{b}$ \\
\hline$y_{10}$ & 3243.465 & 3243.473 \\
\hline$w_{1}$ & 442.016 & 442.099 \\
\hline$w_{2}$ & 747.058 & 747.066 \\
\hline$w_{3}$ & 1076.110 & 1076.135 \\
\hline$w_{4}$ & 1405.163 & 1405.186 \\
\hline$w_{5}$ & 1711.188 & 1711.247 \\
\hline$w_{6}$ & 2040.240 & 2040.407 \\
\hline$w_{7}$ & 2360.286 & 2360.286 \\
\hline$w_{8}$ & 2665.327 & 2665.341 \\
\hline$w_{9}$ & 2994.379 & $2994.102^{b}$ \\
\hline$w_{10}$ & 3323.432 & 3323.408 \\
\hline
\end{tabular}


2) corresponds to methyl-U/ $\Psi$. This was corroborated by a nearly complete pair of ion series $\left(\mathbf{a}-\mathrm{B}\right.$ ase and $\mathbf{d}-\mathrm{H}_{2} \mathrm{O}$; not annotated) (McLuckey et al. 1992) representing the sequence from the $5^{\prime}$ terminus of the T1 fragment. Although a relatively abundant $\mathbf{y}_{6}$ ion was seen, the expected enhancement of the complementary $\mathbf{a}_{5}$ and $\mathbf{w}_{\mathbf{6}}$ ion pair was not observed. This may be a consequence of the difficulty in retaining two and three adenine residues, respectively, on the first-generation dissociation products (McLuckey and Habibi-Goudarzi 1993). Other less abundant ions that arise from multiple dissociation events resulting in scission through the ribose-phosphate backbone and that can be used in further support of the presence of a methyl group at position 1898 are listed in Table 3.

Sequence placement of $\Psi$ at positions 1894 and 1900 was determined by the characteristically elevated responses of $\mathbf{w}$ and $\mathbf{y}$ series ions immediately $3^{\prime}$ of the sites of pseudouridylation (S.C. Pomerantz and J.A. McCloskey, in prep.). The effect is particularly notable in the case of $\Psi$ at the $5^{\prime}$ terminus of the oligonucleotide (ions $\mathbf{w}_{\mathbf{1 0}}$ and $\mathbf{y}_{\mathbf{1 0}}$ ). The significant abundance of ion $\mathrm{m} / \mathrm{z} 165$ reflects the prominence of the dissociation reaction $\mathrm{m} / z 225$ (ion $\mathbf{a}_{1}$ ) $\rightarrow 165$, also a characteristic effect of $\Psi$ at the $5^{\prime}$ position (S.C. Pomerantz and J.A. McCloskey, in prep.). The $\mathbf{a}_{7}$ ion, the $5^{\prime}$ complement to the $\mathbf{w}_{\mathbf{4}}$ ion, did not exhibit any enhanced abundance. This may be reflective of the difficulty in keeping three adenine bases attached to the $\mathbf{a}_{7}$ fragment during fragmentation, as described above.

\section{Sequence localization of the $\Psi$ residues in $H$. marismortui 235 rRNA}

LC/MS/MS screening of an RNase T1 digest of the LSU for $\Psi$ by the multiple reaction monitoring method (data not shown) indicated two principal characteristic responses $(\mathrm{m} / \mathrm{z} 207 \rightarrow 164)$ for oligonucleotides that contained an internal $\Psi$. These oligonucleotides had measured $M_{r}$ values of 1624.95 (5-mer) and 2573.45 (8-mer). The only possible placement of the pentanucleotide within the computer-predicted RNase T1 digest was 2619-UUUAGp, and necessitated the presence of a methyl group ( $M_{r}$ calc. 1624.97). This result was consistent with the reverse transcriptase based finding of $2619-\mathrm{m}^{3} \mathrm{U}$, as described above. LC/MS/MS sequencing of this oligonucleotide (from the $(\mathrm{M}-3 \mathrm{H})^{3-}$ pre-

TABLE 3. Sequence assignments for ions observed in support of $1898-\mathrm{m}^{3} \Psi$

\begin{tabular}{|c|c|}
\hline$m / z$ & Assignment \\
\hline 953.2 & $1896-\mathrm{ACm}^{3} \Psi>p$ or $1897-\mathrm{Cm}^{3} \Psi \mathrm{A}>\mathrm{p}$ \\
\hline 1221.2 & $1898-\mathrm{m}^{3} \Psi \mathrm{A} \Psi \mathrm{A}-\mathrm{OH}$ \\
\hline 1282.2 & $1895-\mathrm{AACm}^{3} \Psi>$ p or $1896-\mathrm{ACm}^{3} \Psi \mathrm{A}>\mathrm{p}$ \\
\hline 1526.2 & $1894-\Psi A A C m^{3} \Psi$ or $1897-\mathrm{Cm}^{3} \Psi A \Psi \mathrm{A}$ \\
\hline 1710.3 & $1898-\mathrm{pm}^{3} \Psi A \Psi A A p$ \\
\hline
\end{tabular}

cursor ion, $60 \mathrm{eV}$ collision $\mathrm{E}_{\mathrm{lab}}$; data not shown) confirmed the CMC-detected presence of $\Psi$ at position 2621 by the enhanced $\mathbf{w}_{\mathbf{2}}$ (64\% relative abundance [RA]) and $\mathbf{y}_{2}(100 \%$ RA) ions, and the complementary $\mathbf{a}_{3}$ ion present at $50 \%$ RA.

The only plausible sequence possibility for the 8-mer in the LSU RNA was 1952-UAACUAUGp $\left(M_{r}\right.$ calc. 2573.56), given the constraints ( $3^{\prime} \mathrm{G}$ terminus, due to use of RNase T1) and mass measurement accuracy of the experiment. This assignment placed the $\Psi$ residue(s) within the structurally conserved helix 69 terminal loop. The LC/MS/MS sequencing spectrum (data not shown) of this oligonucleotide from the $(\mathrm{M}-4 \mathrm{H})^{4-}$ precursor $\left(80 \mathrm{eV}, \mathrm{E}_{\mathrm{lab}}\right)$ did not reveal strong responses at $m / z 225$ and 165 , nor was there an intense $\mathbf{w}_{7}$ ion. These findings precluded the placement of a $\Psi$ at the $5^{\prime}$ terminus (position 1952). Relatively intense responses were observed for the $\mathbf{a}_{5}$ (34\% RA) and $\mathbf{a}_{7}(35 \%$ RA) fragment, and their respective complementary ions $\mathbf{w}_{\mathbf{3}}$ (32\% RA) and $\mathbf{w}_{\mathbf{1}}(72 \% \mathrm{RA})$. The $\mathbf{w}_{\mathbf{1}}$ ion was significantly elevated over the response observed in the $D$. radiodurans loop 69 oligonucleotide (6\% RA). In toto, these data support the RT-based finding of $\Psi$ at positions 1956 and 1958.

\section{Two-dimensional location of $D$. radiodurans and $\boldsymbol{H}$. marismortui $\Psi$}

The $\Psi$ positions in $D$. radiodurans and $H$. marismortui are indicated on secondary structure backbone diagrams of the 23S RNA of the two organisms in Figure 3, panels A and B, respectively. The sites identified in other organisms within the sequences shown are also indicated in the figure. Consistent with all bacteria surveyed so far except E. coli, $\Psi$ in both organisms is only found in the $3^{\prime}$ half of the RNA. As indicated in the figure, none of these $\Psi$ positions are novel. $D$. radiodurans $\Psi 1894,1898$, and 1900 , located in helix 69 , are equivalent to E. coli positions 1911, 1915, and 1917 and are the most conserved $\Psi$ positions among all organisms' cytoplasmic ribosomes from all three phylogenetic kingdoms (Ofengand et al. 2001b). $\Psi$ at position 2584 is equivalent to E. coli position 2605, which is also $\Psi$ in E. coli, Bacillus subtilis, and Zea mays chloroplasts. This single $\Psi$ is made by the $\Psi$ synthase RluB in both E. coli (Del Campo et al. 2001) and B. subtilis (Niu et al. 1999). The $\Psi$ of $H$. marismortui are also not novel. $\Psi 1956$ and 1958 are the aforementioned "most conserved $\Psi$ " that appear in helix 69 and are equivalent to E. coli $\Psi 1915$ and 1917 except that $\Psi 1956$ is not methylated as it is in E. coli. The $\Psi$ at 2621 is a position found in another archaeon, Halobacter halobium, as well as in Drosophila melanogaster and Mus musculus (Ofengand et al. 2001b).

\section{Identification of RluB and RluD homologs in D. radiodurans}

A total of seven $\Psi$ synthase homologs have previously been identified in the genome of $D$. radiodurans (Ofengand et al. 

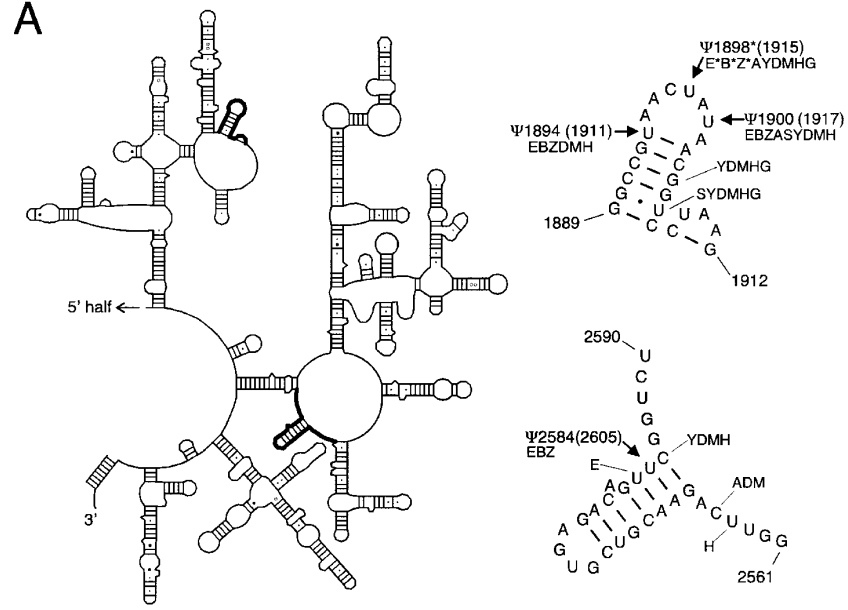

B
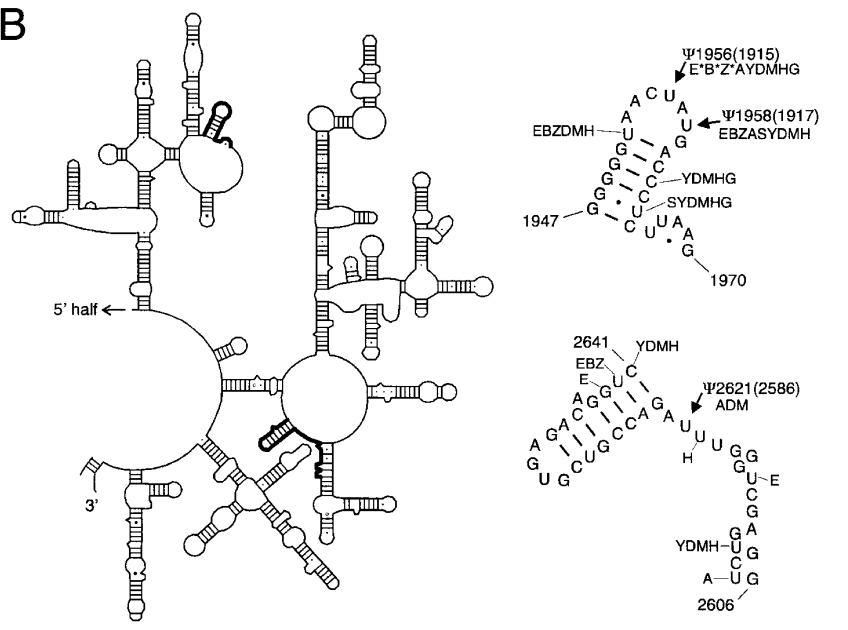

FIGURE 3. Location of $\Psi$ on secondary structures of 23S RNA from $D$. radiodurans $(A)$ and $H$. marismortui $(B)$. In each panel, the secondary structure of the $3^{\prime}$ half of 23S RNA (adapted from the Comparative RNA site, http://www.rna.icmb.utexas.edu) is shown. The regions of the molecule that contain $\Psi$ (bold backbone tracing) are shown in more detail to the right. $\left({ }^{*}\right) \mathrm{m}^{3} \Psi$. Numbering as in Figure 1 . The locations of $\Psi$ in other organisms are as indicated. (E) E. coli; (B) B. subtilis; (Z) Z. mays choloroplasts; (A) H. halobium; (S) S. acidocaldarius; (Y) Saccharomyces cerevisiae; (G) Euglena gracilis; (D) D. melanogaster; (M) M. musculus; (H) H. sapiens (Ofengand 2002; Russell et al. 2004; M.W. Gray, pers. commun.).

2001b; Kaya and Ofengand 2003), but only four belong to the families (RsuA and RluA) known to modify eubacterial rRNA. Among these four, it was expected that D. radiodurans had homologs to E. coli $\mathrm{RluD}$ and RluB since all four $\Psi$ found in D. radiodurans $23 \mathrm{~S}$ RNA are equivalent to $E$. coli $\Psi$ made by these two $\Psi$ synthases. To determine which $E$. coli synthases were most similar to the four D. radiodurans homologs, each E. coli synthase from the RsuA and RluA family was used in a BlastP search against the complete genomic sequence of $D$. radiodurans (Table 4 ). Consistent with previous analyses, only four $D$. radiodurans homologs were identified in this search: one RsuA family member and three RluA family members. The RsuA family member was closest to E. coli RluB and RluF. This is not surprising because RluB and RluF are closely related to each other and target adjacent $U$ residues (Del Campo et al. 2001). Hence, the putative protein gi|15805921| is an RluB homolog. Out of the three RluA family members, the putative protein gi| 15806790 | is clearly the most similar to E. coli RluD and is, therefore, an RluD homolog. For the last two RluA family members, the assignment is unclear. Putative protein gi|15806687| appears to be closest to E. coli RluA, RluC, and RluD, and putative protein gi|15805985| is closest to RluC, RluD, and TruC. The putative protein gi|15806687| likely modifies rRNA because it has an N-terminal S4 domain, a domain thus far found in $\Psi$ synthases that modify rRNA (Del Campo et al. 2004). However, this protein does not appear to be an active synthase since it has a Gly (Gly152; GGG codon) in place of the conserved Asp (GAT or GAC codon) required by $\Psi$ synthases for catalytic activity. Putative protein gi|15805985| has a conserved Asp and is likely to make $\Psi$ in $D$. radiodurans tRNA because all of the rRNA $\Psi_{\mathrm{s}}$ are accounted for by other putative synthase genes.

\section{D. radiodurans has a bona fide RluB and RluD}

After mapping the positions of $D$. radiodurans $\Psi$ and identifying $\Psi$ synthase homologs predicted to form them, the next logical step was to determine whether these $\Psi$ synthase homologs were bona fide $\Psi$ synthases and, if so, which $\Psi$ they made. The most straightforward approach would have been to make gene deletions for the RluB and RluD homologs and to use $\Psi$ sequencing to assess the disappearance of $\Psi$, as was done for $E$. coli. Despite the fact that many genetic tools are becoming available for D. radiodurans, making clean gene deletions is not as straightforward and routine as it is in E. coli. Thus, it seemed more practical to use an approach that took advantage of the E. coli $\Psi$ synthase mutants already in our possession.

If the putative $D$. radiodurans RluB makes $\Psi 2584$ (E. coli $\Psi 2605)$, then it might make $\Psi 2605$ when placed in an $E$. coli strain lacking RluB $(\Delta r l u B)$. Similarly, if the putative $D$. radiodurans $\mathrm{RluD}$ makes $\Psi 1894,1898$, and 1900 , then $D$. radiodurans $\mathrm{RluD}$ might make $\Psi 1911,1915$, and 1917 when placed in an E. coli strain lacking RluD ( $\Delta r l u D:: k a n)$. The D. radiodurans $r l u B$ and $r l u D$ homologs were each cloned into expression vector pTrc99A, transformed into the E. coli $\Delta r l u B$ and $\Delta r l u D:: k a n$ strains, respectively, and grown without induction of the cloned gene. Figure 4A shows that D. radiodurans $\mathrm{RluD}$ is a $\Psi$ synthase able to form $\Psi 1911$ and 1917 in E. coli (the nature of 1915 was discussed above), and, therefore, should be the $\Psi$ synthase responsible for $\Psi 1894,1898$, and 1900 in D. radiodurans. Figure 4B shows that $D$. radiodurans $\mathrm{RluB}$ is a $\Psi$ synthase able to form $\Psi 2605$ in E. coli and, therefore, should be the $\Psi$ synthase responsible for $\Psi 2584$ in D. radiodurans. 
TABLE 4. D. radiodurans homologs of $E$. coli RsuA and RluA family $\Psi$ synthases

\begin{tabular}{|c|c|c|c|c|}
\hline \multirow{2}{*}{$\begin{array}{l}\text { E. coli } \Psi \\
\text { synthase }^{\text {a }}\end{array}$} & \multicolumn{4}{|c|}{ D. radiodurans putative proteins } \\
\hline & gil15805921 $\left.\right|^{\mathrm{b}}$ & gil15806790l & gil15806687| & gil15805985| \\
\hline \multicolumn{5}{|c|}{ RsuA family } \\
\hline RsuA & $5 c^{-19 c}$ & 0.66 & - & - \\
\hline RluB & $2 e^{-31}$ & 4.5 & - & - \\
\hline RluE & $3 e^{-23}$ & 2.3 & 2.3 & - \\
\hline RluF & $1 \mathrm{e}^{-29}$ & 0.003 & - & - \\
\hline \multicolumn{5}{|c|}{ RluA family } \\
\hline RluA & 0.12 & $4 \mathrm{e}^{-26}$ & $3 e^{-22}$ & $1 \mathrm{e}^{-14}$ \\
\hline RluC & 2.9 & $1 e^{-28}$ & $5 e^{-22}$ & $1 \mathrm{e}^{-18}$ \\
\hline RluD & 5.2 & $6 e^{-49}$ & $7 e^{-23}$ & $1 e^{-17}$ \\
\hline TruC & 0.031 & $2 e^{-19}$ & $7 e^{-13}$ & $5 e^{-18}$ \\
\hline
\end{tabular}

${ }^{a}$ Each $E$. coli $\Psi$ synthase from the RsuA and RluA families was used as a query in a BlastP search (low-complexity filtering $=$ off; expect value $\leq 10)$ against only the $D$. radiodurans genomic database (http://www.ncbi.nlm.nih.gov/BLAST).

${ }^{\mathrm{b}}$ Genbank identification numbers.

${ }^{\mathrm{C}}$ Expect values; - indicates $>10$.

\section{DISCUSSION}

\section{Sequencing results}

It was previously reported that there were at least 11 (Ofengand et al. 2001b) or 19 (Ofengand 2002) $\Psi$ located at specific sites in $H$. marismortui $23 \mathrm{~S}$ RNA. These reports were incorrect. We inadvertently failed to completely strip the CMC group from some of the $U$ residues in these experiments, leading us to score those $U$ sites as $\Psi$. We then compounded our error by failing to compare the stop intensity of those $\Psi$ sites with other ones such as the $\Psi 1915$ and $\Psi 1917$ stops. Had we done so, we would have realized that the stops seen were very weak in comparison. We apologize if anyone was misled by these erroneous claims. The error became apparent when the number of $\Psi$ obtained by sequencing was compared with the number from direct nucleoside analysis (Table 1). As a result of this experience, we advise that $\Psi$ sequencing by the CMC-reverse transcriptase method (Ofengand et al. 2001a) always be checked by total nucleoside analysis.

The identification and location of the $\mathrm{m}^{3} \Psi$ residue in $D$. radiodurans $23 \mathrm{~S}$ RNA was carried out by mass spectrometry. Nucleosides from a total nucleoside digest of 16S RNA were analyzed by LC/MS, and no $\mathrm{m}^{3} \Psi$ was detected. A component in the total RNA $(16 \mathrm{~S}+23 \mathrm{~S})$ digest having the correct molecular weight (258), fragmentation behavior, and accurate HPLC retention time for $\mathrm{m}^{3} \Psi$ was observed, which exhibited characteristics identical to $\mathrm{m}^{3} \Psi$, compared in a back-to-back analysis of E. coli $23 S$ RNA. The sequence location was independently confirmed by gas phase sequencing by LC/MS/MS of the expected RNase T1 fragment of composition $\mathrm{U}_{3} \mathrm{C}_{2} \mathrm{~A}_{5} \mathrm{Gp}+\mathrm{CH}_{2}\left(M_{r} 3550.49\right)$ in the RNA ( $\Psi$ and $U$ being indistinguishable in mass). Placements of $\Psi 1894, \mathrm{~m}^{3} \Psi 1898$ and $\Psi 1900$ were based on masses of sequence ions, and abundance changes that are characteristic for pseudouridine and its derivatives (S.C. Pomerantz and J.A. McCloskey, in prep.).

\section{Conservation of the $\Psi$ sites}

Finding three and two $\Psi$ in the helix 69 loop of $D$. radiodurans and $H$. marismortui, respectively, was not surprising given the high conservation of these modification sites. As shown in Table 5, position 1917 is almost always $\Psi$, at least in the 12 organisms so far sequenced in this area, and excluding known mitochondrial ribosomes that have no $\Psi$ in this stem-loop. Position 1915 is also $\Psi$ or $\mathrm{m}^{3} \Psi$ in all the organisms except Sulfolobus acidocaldarius despite the presence of U1915 in that organism. As archaea likely make their ribosomal $\Psi$ using guide RNAs for specifying the sites rather than an RluD-like enzyme, it appears that the needed guide RNA is absent in S. acidocaldarius. Position 1911 is more variable, being $\Psi$ in all four of the eubacteria and eubacteria-derived organelles, $U$ in the three archaea, and either $\mathrm{U}$ or $\Psi$ in the eukaryotes. Thus, one

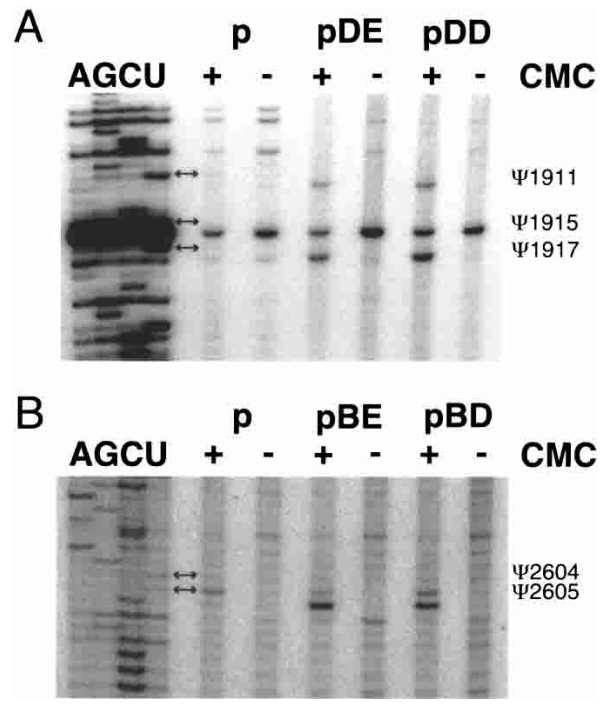

FIGURE 4. $\Psi$ sequencing of 23 SNA from E. coli $\Psi$ synthase mutants complemented with putative $\Psi$ synthases from $D$. radiodurans. (A) The E. coli $\Psi$ synthase mutant $\Delta r l u D$ transformed with plasmid pTrc99A carrying no insert (p) or RluD from E. coli (pDE) or $D$. radiodurans (pDD). (B) The E. coli $\Psi$ synthase mutant $\Delta r l u B:: k a n$ transformed with plasmid pTrc99A carrying no insert (p) or RluB from E. coli (pBE) or D. radiodurans (pBD). In both panels, $\Psi$ are indicated by $E$. coli numbering and RNA sequence is shown in A, C, $\mathrm{G}$, and $\mathrm{U}$ lanes. Transformation, growth of uninduced transformed cells, and isolation of total RNA was done as described (Del Campo et al. 2001). 
striking finding of this work is that the conservation of $\Psi$ sites in the loop of helix 69 persists in the two diverse organisms, $D$. radiodurans and $H$. marismortui.

We did not expect to find that, with the exception of helix 69 , there would be only a single $\Psi$ in the entire $23 \mathrm{~S}$ RNA of both organisms because analysis of other bacteria has generally found more (Ofengand et al. 2001b). Our original hope had been that enough $\Psi$ would be found in sufficiently diverse locations that knowing their three-dimensional position in the ribosome would enable some assessment of their functional and/or structural role. On the other hand, the fact that there is only one additional $\Psi$ in each organism, albeit a different one in the two cases, implies that there is some specific importance to these two sites. At the 2586 site, the eubacteria lack $\Psi$ even though $E$. coli has a U2586. H. marismortui and H. halobium as well as three eukaryotes have $\Psi 2586$ (2585 in Homo sapiens). Curiously, yeast does not have $\Psi$ there despite having U2586. The $D$. radiodurans $\Psi 2605$ is also present in the other eubacteria but is absent from $H$. marismortui, H. halobium, and the eukaryotes despite the presence of the precursor $\mathrm{U}$ at that site. Instead, the eukaryotes have $\Psi 2606$, which is not possible in the archaea or eubacteria because the position is occupied by a C.

The first conclusion to be made from this analysis is that $\Psi$ at the apex of helix 69 is necessary, although the exact number is subject to some variation. The second conclusion is that there is some ill-defined connection between $\Psi 2586$ and $\Psi 2605-2606$. Since U2605 is available in all the eukaryotes studied but is not modified, $\Psi 2606$ is likely to be the preferred site of modification. It is not available in the eubacteria and archaea that were sequenced for $\Psi$, and in fact this residue is $\mathrm{C}$ in $>80 \%$ of known archaeal and eubacterial 23S RNA sequences (Comparative RNA site, http://www. rna.icmb.utexas.edu). Eubacteria appear to use the adjacent residue, $\Psi 2605$, as a substitute (E. coli has $\Psi 2604$ in addition) whereas in the two archaeons studied, U2605 is not used but instead U2586 is converted to $\Psi$. Interestingly, eukaryotes with the exception of yeast make $\Psi$ at both U2606 and U2585, perhaps to achieve a stronger effect of whatever it is that $\Psi$ does.

\section{Potential roles for the $\Psi$ residues identified in this work}

The most important question about $\Psi$ in rRNA is what they do that $U$ cannot. This is still an unanswered question. Nevertheless, there are intriguing correlations between the locations of the $\Psi$ residues and nucleosides known to be important for ribosome function. In the $70 \mathrm{~S}$ ribosometRNA structure (Yusupov et al. 2001), the loop of helix 69 containing the $\Psi$ supports the anticodon arm of A-site tRNA near its juncture with the amino acid arm. The middle of helix 69 does the same thing for P-site tRNA. Unfortunately, the resolution in this work was not sufficient to reveal any specific roles for the $\Psi$ residues. On the other hand, a recent study of the location of the ribosome release factor RRF on the 70S ribosome has shown that $\mathrm{m}^{3} \Psi 1915$ makes a direct contact with amino acid residues E122 and V126 of the ribosome release factor RRF (Agrawal et al. 2004). It may therefore turn out that the primary protein synthesis functional role for $\Psi$ in helix 69 is in ribosome release rather than in the more conventional aspects of protein synthesis.

To see if the putative connection between $\Psi 2605$ and $\Psi 2586$ (E. coli numbering) based on conservation (Table 5) has any structural basis, the environment around $D$. radiodurans (PDB no. 1NKW) $\Psi 2584$ and C2565 (E. coli 2605

TABLE 5. Species conservation of $\Psi$ at or near the sites found in $H$. marismortui and D. radiodurans

\begin{tabular}{|c|c|c|c|c|c|c|c|c|c|}
\hline \multirow[b]{2}{*}{ Class } & \multirow[b]{2}{*}{ Organism } & \multicolumn{8}{|c|}{ Position (Escherichia coli numbering) } \\
\hline & & 1911 & 1915 & 1917 & 2585 & 2586 & 2604 & 2605 & 2606 \\
\hline \multirow[t]{4}{*}{ Eubacteria, chloroplasts } & Escherichia coli & $\Psi$ & $\mathrm{m}^{3} \Psi$ & $\Psi$ & $U$ & $U$ & $\Psi$ & $\Psi$ & $\mathrm{C}$ \\
\hline & Bacillus subtilis & $\Psi$ & $\Psi^{*}$ & $\Psi$ & U & C & $\cup$ & $\Psi$ & C \\
\hline & Deinococcus radiodurans & $\Psi$ & $\mathbf{m}^{3} \Psi$ & $\Psi$ & $\mathbf{U}$ & C & $\mathbf{U}$ & $\Psi$ & C \\
\hline & Zea mays chloroplasts & $\Psi$ & $\Psi^{*}$ & $\Psi$ & U & C & U & $\Psi$ & C \\
\hline \multirow[t]{3}{*}{ Archaea } & Halobacter halobium & U & $\Psi$ & $\Psi$ & $U$ & $\Psi$ & G & U & $\mathrm{C}$ \\
\hline & Haloarcula marismortui & $\mathbf{U}$ & $\Psi$ & $\Psi$ & $\mathbf{U}$ & $\Psi$ & G & $\mathbf{U}$ & C \\
\hline & Sulfolobus acidocaldarius & U & U & $\Psi$ & $U^{a}$ & $U^{\mathrm{a}}$ & G & $U^{\mathrm{a}}$ & C \\
\hline \multirow[t]{5}{*}{ Eukarya } & Saccharomyces serevisiae & U & $\Psi$ & $\Psi$ & $U$ & $U$ & $G$ & $U$ & $\Psi$ \\
\hline & Euglena gracilis & $U$ & $\Psi$ & $U$ & $U^{a}$ & $U^{a}$ & G & $U^{a}$ & $U^{a}$ \\
\hline & Drosophila melanogaster & $\Psi$ & $\Psi$ & $\Psi$ & $U$ & $\Psi$ & $\mathrm{G}$ & $U$ & $\Psi$ \\
\hline & Mus musculus & $\Psi$ & $\Psi$ & $\Psi$ & $U$ & $\Psi$ & $G$ & U & $\Psi$ \\
\hline & Homo sapiens & $\Psi$ & $\Psi$ & $\Psi$ & $\Psi$ & $U$ & G & $U$ & $\Psi$ \\
\hline
\end{tabular}


and 2586, respectively) and around H. marismortui (PDB no. 1S72) $\Psi 2621$ and U2640 (E. coli 2586 and 2605, respectively) was examined. Although the $\mathrm{N}_{1}$ position of the equivalent residues are only about $14 \AA$ apart when both $\Psi$ are placed in either structure, the $\mathrm{N}_{1}-\mathrm{H}$ bonds do not point in a common direction nor do they interact with any shared in common component of the ribosome or its bound tRNA analogs at $\mathrm{A}, \mathrm{P}$, or $\mathrm{E}$ sites (PDB nos. 1S72, 1FFZ, 1KQS, 1M9O, 1QVF, $1 \mathrm{NJM}, 1 \mathrm{NJO}$, and $1 \mathrm{NJP}$ were examined). $H$. marismortui $\Psi 2621$ is at the tunnel entrance but in $D$. radiodurans $\Psi 2584$ is not, and there are no apparent ribosomal components in position to interact with either $\Psi$. There is, on the other hand, a certain "always a bridesmaid never a bride" quality to these two $\Psi$, a property noted also in other $\Psi$ in rRNA (Ofengand et al. 2001b; Ofengand 2002). For example, H. marismortui $\Psi 2621$ is adjacent to the universally conserved U2620, which makes contact with tRNA analogs in the 50S structure (PDB no. 1KQS). Likewise, D. radiodurans $\Psi 2584$ is only three residues removed from A2581, a virtually universal residue that is bulged out from the tunnel wall, and is thought to provide half of the double anchor for the A- to P-site spiral motion proposed by the group of Yonath (Agmon et al. 2004). Thus $\Psi$ residues continue to be near, but not exactly at, nucleotides directly involved in ribosome function.

\section{$\Psi$ synthases in $D$. radiodurans}

We identified two $\Psi$ synthases, RluB and RluD, most likely responsible for $\Psi$ s 2584 and 1894, 1898, and 1900, respectively, in $D$. radiodurans 23 SNA by combining our knowledge of E. coli $\Psi$ sites and synthases with bioinformatics tools. While definitive assignment can only be done by performing the genetic analysis in $D$. radiodurans, our complementation studies of $E$. coli mutants clearly show that $D$. radiodurans $\mathrm{RluB}$ and $\mathrm{RluD}$ have $\Psi$ synthase activity with site capability identical to E. coli RluB and RluD, respectively. Of the two remaining $D$. radiodurans homologs that could also make these rRNA $\Psi$ s if one assumes overlapping site specificity, one (gi|15806687|) appears to be an inactive $\Psi$ synthase lacking the conserved catalytic Asp. It would take two base changes to obtain the conserved Asp from the Gly codon (GGG) at the active site, so this is not likely a DNA sequencing error. Perhaps this synthase has lost its capacity to make $\Psi$ but has another function in D. radiodurans. The other homolog (gi|15805985|) might share site specificity with either RluB or RluD, but it is more likely that this $\Psi$ synthase is specific for tRNA since bacterial $\Psi$ synthases do not appear to have overlapping specificity.

\section{MATERIALS AND METHODS}

\section{Strains and growth conditions}

D. radiodurans R1 (a gift from Michael J. Daly, Uniformed Services Univ. of the Health Sciences) was grown in TGY medium (0.5\% Bacto-tryptone, $0.1 \%$ glucose, $0.3 \%$ Bacto-yeast extract) at $30^{\circ}$ C. H. marismortui (a gift from Peter Moore, Yale University, who also supplied an initial sample of ribosomes) was grown in a modified ATCC medium 1230 at $37^{\circ} \mathrm{C}$. The medium contained 25 $\mathrm{mM} \mathrm{KCl}, 76 \mathrm{mM} \mathrm{MgSO}_{4}, 11 \mathrm{mM} \mathrm{Na}$ citrate, $4 \mathrm{M} \mathrm{NaCl}, 1.7 \mathrm{mM}$ $\mathrm{CaCl}_{2}, 5 \mathrm{~g} / \mathrm{L}$ yeast extract (Difco), $47 \mathrm{mM}$ Tris- $\mathrm{HCl}$ (pH 7.2), $4 \mathrm{~g} / \mathrm{L}$ glucose, $0.01 \mathrm{mM} \mathrm{MnCl}$, and $1.7 \mathrm{mM} \mathrm{FeCl}_{3}$ adjusted to $\mathrm{pH}$ 7.2-7.4. The following E. coli strains were used: MG1655 (wildtype E. coli K12), MG1655 $\Delta r l u B$ (Del Campo et al. 2001), and MG1655 $\Delta$ rluD::kan (N. Gutgsell and J. Ofengand, unpubl. results). E. coli strains were grown at $37^{\circ} \mathrm{C}$ in $\mathrm{LB}$ medium $(1 \%$ Bacto-tryptone, $1 \% \mathrm{NaCl}, 0.5 \%$ Bacto-yeast extract) supplemented with carbenicillin $(100 \mu \mathrm{g} / \mathrm{mL})$ for plasmid selection. In addition, kanamycin $(30 \mu \mathrm{g} / \mathrm{mL})$ was used when growing the $\Delta r l u D:: k a n$ strain.

\section{RNA isolation and $\Psi$ sequencing}

E. coli and $H$. marismortui total RNA were isolated from cells grown to an $\mathrm{A}_{600}$ of $1-1.5$ at $37^{\circ} \mathrm{C}$, following the procedure described by Ofengand et al. (2001a). Total $D$. radiodurans $\mathrm{rRNA}$ was obtained from cells grown to an $\mathrm{A}_{600}$ of 0.9 , and isolated using the RNeasy kit (Qiagen) according to the manufacturer's instructions. This latter procedure removes small MW RNAs such as 5S RNA but does not separate the $16 \mathrm{~S}$ and $23 \mathrm{~S}$ RNAs. E. coli and H. marismortui total RNA were fractionated into $16 \mathrm{~S}$ and 23S RNAs by sucrose gradient centrifugation. The RNA, dissolved in $20 \mathrm{mM}$ HEPES (pH 7.5), $100 \mathrm{mM} \mathrm{LiCl,} 2$ mM EDTA, 0.1\% SDS, was run on $10 \%-25 \%$ sucrose gradients in the same buffer at $22,000 \mathrm{rpm}$ for $30 \mathrm{~h}$ at $17^{\circ} \mathrm{C}$ in a Beckman SW28 rotor. Fractions were collected and the size and purity examined by glyoxal- $\mathrm{Me}_{2} \mathrm{SO}$ denaturing gel electrophoresis (Denman et al. 1989). D. radiodurans $16 \mathrm{~S}$ and $23 \mathrm{~S}$ RNA was obtained by first isolating $30 \mathrm{~S}$ and $50 \mathrm{~S}$ ribosomal subunits on sucrose gradients and then extracting the subunits with phenol/chloroform to obtain the RNA. This procedure was followed because the 23S RNA in 50S subunits has cleavages that would result in contamination of the 16S RNA if it were isolated by the same procedure used for the other rRNAs. $D$. radiodurans ribosomal subunits were obtained as follows. Cells were washed in buffer A (10 mM HEPES at pH 7.8, 4 mM mercaptoethanol, $150 \mathrm{mM} \mathrm{NH}_{4} \mathrm{Cl}, 30 \mathrm{mM} \mathrm{Mg}(\mathrm{OAc})_{2}$ ) and then suspended in buffer $A$ at $125 \mathrm{mg} / \mathrm{mL}$ and treated with $0.25 \mu \mathrm{g} / \mathrm{mL}$ lysozyme for $20 \mathrm{~min}$ at $0^{\circ} \mathrm{C}$ with shaking. The mixture was then passed through a French press at 600-650 bars, RNase-free DNase was added at $0.1 \mu \mathrm{g} / \mathrm{mL}$, and the mixture incubated at $0^{\circ} \mathrm{C}$ for 30 min. Cell debris was removed by centrifugation for $20 \mathrm{~min}$ at $13,000 \mathrm{rpm}$ in a Sorvall SS-1 rotor. Per volume of supernatant, 0.2 volumes of $1.1 \mathrm{M}$ sucrose in buffer A was added and centrifuged $3 \mathrm{~h}$ at 19,000 rpm in a Beckman 45Ti rotor. This supernatant was layered over a cushion of $1.1 \mathrm{M}$ sucrose in buffer A and centrifuged (60Ti rotor, 42,000 rpm, $22 \mathrm{~h}$ ). The pellet was resuspended in buffer $\mathrm{A}$ and layered on a sucrose gradient in $10 \mathrm{mM}$ HEPES (pH 7.8), $4 \mathrm{mM}$ mercaptoethanol, $100 \mathrm{mM} \mathrm{NH}_{4} \mathrm{Cl}, 5-6 \mathrm{mM}$ $\mathrm{Mg}(\mathrm{OAc})_{2}$ and centrifuged to obtain the separated subunits. All RNAs were sequenced for $\Psi$ as previously described (Ofengand et al. 2001a) except that the alkaline treatment was done for 4 and 6 $\mathrm{h}$ to better assess CMC removal from $\mathrm{U}$ and $\mathrm{G}$ residues.

\section{Cloning $D$. radiodurans RluB and RluD homologs}

The D. radiodurans genes rluB (gi|15805921|) and rluD (gi|15806790|), whose gene products are homologs of E. coli RluB 
and RluD, respectively, were PCR amplified from D. radiodurans R1 genomic DNA, subcloned into the pGEM-T vector (Promega), and then cloned into the NcoI and HindIII sites of the pTrc99A expression vector. For the $774 \mathrm{bp} r l u B$, the $\mathrm{N}$-terminal primer (5'-CTAGAAGACTTCATGTCCGCTGAGCGCTTGCA-3') incorporated a BbsI site (italics) at the fourth nucleotide preceding the AUG start codon (underlined) and the C-terminal primer (5' AATGAAGCTTGCAGAAGGCGCTGTGAGGC-3') incorporated a HindIII site (italics) at the 26th nucleotide following the TAA stop codon. For the 1017 bp $r l u D$, the N-terminal primer ( $5^{\prime}$ AATAGGTCTCACATGCCACGTTGGCCGGAACAG-3') incorporated a BsaI site (italics) at the third nucleotide preceding the AUG start codon (underlined) and the C-terminal primer ( $5^{\prime}$ TGTCAAGCTTCCACCAGCGGAGAGGGTCAA- $3^{\prime}$ ) incorporated a HindIII site (italics) at the 50th nucleotide following the TAG stop codon. Both constructs were verified by DNA sequencing.

\section{Nucleoside analysis}

Individual 16S and 23S RNAs were digested to nucleosides with RNase P1, venom phosphodiesterase, and alkaline phosphatase as described (Crain 1990). The digests were analyzed by directly combined LC/MS in positive ion mode on a Waters Quattro II mass spectrometer fitted with the Z-spray electrospray ion source coupled to an Agilent Model 1090 liquid chromatograph. Quantitation was obtained from LC peak areas, the mass spectral data being used to verify LC peak identity and purity. Components of the digest were resolved on a Phenomenex Luna C18 (Crain 1990) column $\left(2.1 \times 250 \mathrm{~mm}, 5 \mu \mathrm{d}_{\mathrm{p}}\right)$ protected by a Opti-Guard precolumn (Optimize Technologies). Buffer A was $5 \mathrm{mM} \mathrm{NH}_{4} \mathrm{OAc}$ ( $\mathrm{pH} 5.3$ ) and buffer $\mathrm{B}$ was $40 \% \mathrm{CH}_{3} \mathrm{CN}$. A multilinear gradient was employed (Pomerantz and McCloskey 1990) and the total liquid flow of the LC $(300 \mu \mathrm{L} / \mathrm{min})$ was analyzed. Typically, full scan mass spectra were acquired from $\mathrm{m} / z$ 108-408 every $0.45 \mathrm{sec}$. The presence of $\mathrm{m}^{3} \Psi$ was confirmed in the combined 16S and 23S RNA digest by selected ion recording (SIR) data acquisition of the $m / z 259\left(\mathrm{MH}^{+}\right)$and $223\left(\mathrm{MH}^{+}-2 \mathrm{H}_{2} \mathrm{O}\right)$ channels with a 90-msec dwell time. Quantitation of $\Psi$ was accomplished by integration of the 260-nm peak areas from the HPLC diode array data by the MassLynx data system without data smoothing. The peak areas were calculated for $\Psi$ and $\mathrm{C}$, and then corrected for the empirically determined (in the McCloskey laboratory) relative molar response ratio at $260 \mathrm{~nm}$. Since the number of $\mathrm{C}$ residues is known (from the gene sequence), simple multiplication allowed calculation of the number of $\Psi$ residues.

\section{Oligonucleotide analysis}

RNAs were digested to oligonucleotides with 10 units of RNase T1 (Ambion) per pmol of RNA in Tris-EDTA buffer (pH 7.0) (Crain 1990). Oligonucleotides were chromatographically resolved on Luna C18 (Crain 1990) columns (Phenomenex) using $400 \mathrm{mM}$ hexafluoroisopropanol (HFIP) titrated to $\mathrm{pH} 7$ with triethylamine and a linear gradient (1\%/min) against $400 \mathrm{mM} \mathrm{HFIP} \mathrm{in} 50 \%$ methanol (Apffel et al. 1997). For molecular weight determination and testing for the presence of $\Psi$, a $1.0 \times 150 \mathrm{~mm}$ column at a flow rate of $60 \mu \mathrm{L} / \mathrm{min}$ was used (Agilent 1090). Component resolution for de novo sequencing of $\Psi$-containing oligonucleotides was per- formed on a $0.5 \times 150 \mathrm{~mm}$ column at a flow rate of $15 \mu \mathrm{L} / \mathrm{min}$. The presence of $\Psi$ was detected by a reaction-monitoring experiment performed on a Waters Quattro II instrument in which the $\mathrm{m} / \mathrm{z} 207$ ion, equivalent to the doubly dehydrated nucleoside anion, was generated by elevating the cone voltage of the ion source to $70 \mathrm{~V}$, from its normal $35 \mathrm{~V}$ used for molecular weight determination. The $\mathrm{m} / z 207$ ion was selected in the first quadrupole, dissociated at $14 \mathrm{eV}$ at an argon gas pressure of $3 \times 10^{-3} \mathrm{mbar}$ in the collision cell, and the product ion at $\mathrm{m} / \mathrm{z} 164$ was monitored in the third quadrupole with a dwell time of $30 \mathrm{msec}$ (S.C. Pomerantz and J.A. McCloskey, in prep.). De novo sequencing was accomplished with a Waters CapLC chromatograph coupled to a Micromass Q-Tof 2 mass spectrometer. The $(\mathrm{M}-6 \mathrm{H})^{6-}$ ion was selected in the quadrupole, and the product ions were measured using the time-of-flight analyzer after dissociation at $90 \mathrm{eV}$ (laboratory frame of reference) collision energy.

\section{ACKNOWLEDGMENTS}

We dedicate this paper to the vibrant life and scientific career of Professor James Ofengand. Jim was a model scientist, orator, and mentor. He was also a great friend and colleague. He left us all too soon and we will dearly miss him.

We thank Dr. Francois Franceschi for the procedure used to obtain $D$. radiodurans ribosomal subunits. This work was supported in part by NIH grants GM58879 (J.O.) and GM29812 (J.A.M), and NIH fellowship GM66374 (M.D).

Received October 18, 2004; accepted November 19, 2004.

\section{REFERENCES}

Agmon, I., Amit, M., Auerbach, T., Bashan, A., Baram, D., Bartels, H., Berisio, R., Greenberg, I., Harms, J., Hansen, H.A., et al. 2004. Ribosomal crystallography: A flexible nucleotide anchoring tRNA translocation, facilitates peptide-bond formation, chirality discrimination and antibiotics synergism. FEBS Lett. 567: 20-26.

Agrawal, R.K., Sharma, M.R., Kiel, M.C., Hirokawa, G., Booth, T.M., Spahn, C.M.T., Grassucci, R.A., Kaji, A., and Frank, J. 2004. Visualization of ribosome-recycling factor on the Escherichia coli $70 \mathrm{~S}$ ribosome: Functional implications. Proc. Natl. Acad. Sci. 101: 8900-8905.

Apffel, A., Chakel, J.A., Fischer, S., Lichtenwalter, K., and Hancock, W.S. 1997. Analysis of oligonucleotides by HPLC-electrospray ionization mass spectrometry. Anal. Chem. 69: 1320-1325.

Ban, N., Nissen, P., Hansen, J., Moore, P.B., and Steitz, T.A. 2000. The complete atomic structure of the large ribosomal subunit at $2.4 \AA$ resolution. Science 289: 905-920.

Cohn, W.E. 1960. Pseudouridine, a carbon-carbon linked ribonucleoside in ribonucleic acids: Isolation, structure, and chemical characteristics. J. Biol. Chem. 235: 1488-1498.

Crain, P.F. 1990. Preparation and enzymatic digestion of RNA and DNA for mass spectrometry. Methods Enzymol. 193: 782-790.

Del Campo, M., Kaya, Y., and Ofengand, J. 2001. Identification and site of action of the remaining four putative pseudouridine synthases in Escherichia coli. RNA 7: 1603-1615.

Del Campo, M., Ofengand, J., and Malhotra, A. 2004. Crystal structure of the catalytic domain of RluD, the only rRNA pseudouridine synthase required for normal growth of Escherichia coli. RNA 10: 231-239.

Denman, R., Weitzmann, C., Cunningham, P.R., Negre, D., Nurse, K., Colgan, J., Pan, Y.C., Miedel, M., and Ofengand, J. 1989. In vitro assembly of $30 \mathrm{~S}$ and $70 \mathrm{~S}$ bacterial ribosomes from 16S RNA con- 
taining single base substitutions, insertions, and deletions around the decoding site (C1400). Biochemistry 28: 1002-1011.

Felden, B., Hanawa, K., Atkins, J.F., Himeno, H., Muto, A., Gesteland, R.F., McClsokey, J.A., and Crain, P.F. 1998. Presence and location of modified nucleotides in Escherichia coli tmRNA: Structural mimicry with tRNA isoacceptor branches. EMBO J. 17: 3188-3196.

Gehrke, C.W. and Kuo, K.C. 1990. Ribonucleoside analysis by reversed-phase high performance liquid chromatography. In Chromatography and identification of nucleosides, part A, Journal of chromatography library (eds. C.W. Gehrke and K.C. Kuo), pp. A3-A64. Elsevier, New York.

Hansen, M.A., Kirpekar, F., Ritterbusch, W., and Vester, B. 2002. Posttranscriptional modifications in the A-loop of $23 \mathrm{~S}$ rRNAs from selected archaea and eubacteria. RNA 8: 202-213.

Harms, J., Schluenzen, F., Zarivach, R., Bashan, A., Gat, S., Agmon, I., Bartels, H., Franceschi, F., and Yonath, A. 2001. High resolution structure of the large ribosomal subunit from a mesophilic eubacterium. Cell 107: 679-688.

Kaya, Y. and Ofengand, J. 2003. A novel unanticipated type of pseudouridine synthase with homologs in bacteria, archaea, and eukarya. RNA 9: 711-721.

Klein, D.J., Moore, P.B., and Steitz, T.A. 2004. The roles of ribosomal proteins in the structure, assembly, and evolution of the large ribosomal subunit. J. Mol. Biol. 340: 141-177.

Kowalak, J.A., Bruenger, E., Hasizume, T., Peltier, J.M., Ofengand, J., and McCloskey, J.A. 1996. Structural characterization of $U^{\star}-1915$ in domain IV from Escherichia coli $23 \mathrm{~S}$ ribosomal RNA as 3-methylpseudouridine. Nucleic Acids Res. 24: 688-693.

Maden, B.E.H. 1990. The numerous modified nucleotides in eukaryotic ribosomal RNA. Progr. Nucleic Acids Res. Mol. Biol. 39: 241300.

McLuckey, S.A. and Habibi-Goudarzi, S. 1993. Decompositions of multiply charged oligonucleotide anions. J. Am. Chem. Soc. 115: 12085-12095.

McLuckey, S.A., Van Berkel, G.J., and Glish, G.L. 1992. Tandem mass spectrometry of small, multiply charged oligonucleotides. J. Am. Soc. Mass Spectrom. 3: 60-70.

Ni, J., Pomerantz, S.C., Rozenski, J., Zhang, Y., and McCloskey, J.A. 1996. Interpretation of oligonucleotide mass spectra for determination of sequence using electrospray ionization and tandem mass spectrometry. Anal. Chem. 68: 1989-1999.

Niu, L., Lane, B.G., and Ofengand, J. 1999. Cloning and characterization of the 23S RNA pseudouridine 2633 synthase from Bacillus subtilis. Biochemistry 38: 629-635.

Noon, K.R., Bruenger, E., and McCloskey, J.A. 1998. Posttranscriptional modifications in $16 \mathrm{~S}$ and $23 \mathrm{~S}$ rRNAs of the archaeal hyperthermophile Sulfolobus solfataricus. J. Bacteriol. 180: 2883-2888.

Ofengand, J. 2002. Ribosomal RNA pseudouridines and pseudouridine synthases. FEBS Lett. 514: 17-25.

Ofengand, J. and Bakin, A. 1997. Mapping to nucleotide resolution of pseudouridine residues in large subunit ribosomal RNAs from representative eukaryotes, prokaryotes, archaebacteria, mitochondria, and chloroplasts. J. Mol. Biol. 266: 246-268.

Ofengand, J., Del Campo, M., and Kaya, Y. 2001a. Mapping pseudouridines in RNA molecules. Methods 25: 365-373.

Ofengand, J., Malhotra, A., Remme, J., Gutgsell, N.S., Del Campo, M., Jean-Charles, S., Peil, L., and Kaya, Y. 2001b. Pseudouridines and pseudouridine synthases of the ribosome. Cold Spring Harbor Symp. Quant. Biol. 66: 147-159.

Pomerantz, S.C. and McCloskey, J.A. 1990. Analysis of RNA hydrolyzates by LC/MS. Methods Enzymol. 193: 796-824.

Raychaudhuri, S., Conrad, J., Hall, B.G., and Ofengand, J. 1998. A pseudouridine synthase required for the formation of two universally conserved pseudouridines in ribosomal RNA is essential for normal growth of Escherichia coli. RNA 4: 1407-1417.

Rozenski, J., Crain, P.F., and McCloskey, J.A. 1999. The RNA modification database: 1999 update. Nucleic Acids Res. 27: 196-197.

Russell, A.G., Schnare, M.N., and Gray, M.W. 2004. Pseudouridineguide RNAs and other Cbf5p-associated RNAs in Euglena gracilis. RNA 10: 1034-1046.

Sprinzl, M. and Vassilenko, K.S. 2002. Compilation of tRNA sequences and sequences of tRNA genes. http://www.uni-bayreuth. de/departments/biochemie/trna/.

Yusupov, M.M., Yusupova, G.Z., Baucom, A., Lieberman, K., Earnest, T.N., Cate, J.H.D., and Noller, H.F. 2001. Crystal structure of the ribosome at $5.5 \AA$ resolution. Science 292: 883-896.

Zamir, A., Holley, R.W., and Marquisee, M. 1965. Evidence for the occurrence of a common pentanucleotide sequence in the structures of transfer ribonucleic acids. J. Biol. Chem. 240: 12671273. 

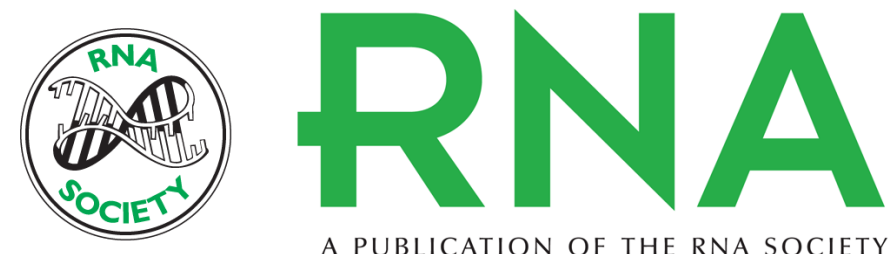

A PUBLICATION OF THE RNA SOCIETY

\section{Number, position, and significance of the pseudouridines in the large subunit ribosomal RNA of Haloarcula marismortui and Deinococcus radiodurans}

MARK DEL CAMPO, CLAUDIA RECINOS, GISCARD YANEZ, et al.

RNA 2005 11: 210-219

References This article cites 31 articles, 13 of which can be accessed free at:

http://rnajournal.cshlp.org/content/11/2/210.full.html\#ref-list-1

License

Email Alerting Receive free email alerts when new articles cite this article - sign up in the box at the

Service top right corner of the article or click here. 